\title{
Pilot study of Stem Cell Therapy in Heart Failure Patients
}

\author{
S M MUSTAFAZAMAN ${ }^{1}$, MD. FAKHRUL ISLAM KHALED ${ }^{1}$, BISHNU PADA DEY ${ }^{2}$, MASUDA BEGUM ${ }^{3}$, SAYED \\ MAINUDDIN AHMED ${ }^{4}$, MD. AZHARUL ISLAM ${ }^{1}$, SAJAL KRISHNA BANERJEE ${ }^{1}$, MD. HARISUL HOQUE ${ }^{1}$ \\ ${ }^{1}$ Department of Cardiology, Bangabandhu Sheikh Mujib Medical University (BSMMU), Dhaka, ${ }^{2}$ Department of Pathology, \\ BSMMU, Dhaka, ${ }^{3}$ Department of Haematology, BSMMU, Dhaka, ${ }^{4}$ Gopalgonj Sadar Hospital, Gopalgonj
}

\begin{abstract}
:
Introduction: Chronic heart failure with reduced ejection fraction is a major complication of diseases involving myocardium. Despite numerous pharmacological interventions and invasive therapeutic techniques, therapeutic options for end stage heart failure remain limited to left ventricular assist device \& organ transplantation. Regenerative medicine may bring hope here. Method: This pilot study was carried out at the Department of cardiology in collaboration with department of haematology, Bangabandhu Sheikh Mujib Medical University, Dhaka, from October 2017 to March 2018. Considering inclusion \& exclusion crieteria ten (10) patients were taken in stem cell group and ten (10) patients in control group. Patients in the control arm received standard of care in accordance with practice guidelines for heart failure management (GDMT). Patients in the cell therapy arm received, in addition to standard of care, bone marrow-derived cardiopoietic stem cells (G-CSF) meeting quality release criteria. Baseline clinical and echocardiographic data were obtained and recorded in pre-formed data sheet. Close liaison was maintained with all patients and followed up after 30 days \& after 3 months and for any complication. The absolute change in 6 MWD from baseline to 30 days, 3 months \& 6 months improved significantly in the both groups. But significant improvement was found at 6 months follow up of 6 MWD between the two groups $(300 \pm 28$ vs $375 \pm 25, p=0.04)$. Baseline BORG scale was similar in the control group and the $S C T$ group $(8.1 \pm 0.56$ and $8.3 \pm 0.67$ respectively, $P=0.45)$. The absolute change in BORG scale from baseline to 30 days, 3 months \& 6 months improved significantly in the both groups. But improvement was not statistically significant in between the two groups $(p=0.32,0.45,0.23$ respectively). Echocardiographic observation also revealed a similar baseline LVIDd, LVEF level in the control group and the SCT group which was not statistically significant $(p=0.45,0.52$ respectively). Gradual improvement in LVIDd were found at 30 days, 3 months, 6 months follow up observation but statistically significant absolute change was found only at 6 months follow up in between groups (62.4 1.8 vs 56 \pm 2.4 , p=0.03). Baseline LVEF were less than $30 \%$ in both control \& SCT group $(29.5 \pm 0.8 \%$ \& $28.7 \pm 1.3 \%$ respectively). The echocardiographic evaluation also revealed a significant increase in LVEF at 6 months $(34 \% \pm 1.6$ and $40 \% \pm 2.5 \%, p=0.04)$ of follow-up in between group but not at 30 days \& 3 months follow up.
\end{abstract}

Key words: Heart failure, Stem cell therapy.

University Heart Journal 2020; 16(2): 52-58

Introduction:

Patients having coronary artery disease, valvular heart disease, myocardial \& other cardiac disorders are at risk of development of heart failure with reduced ejection fraction. Despite numerous pharmacological interventions and invasive therapeutic techniques, therapeutic options for end stage heart failure remain limited to left ventricular assist device \& organ transplantation. So, the patients \& physicians are seeking an alternative treatment strategy. Now a days regenerative medicine is getting interest in end stage organ dysfunctions.

The pathology of heart failures include intrinsic defects of the contractility of cardiac muscle due to the molecular pathology, defect in energy production; extrinsic defects to cardiac muscle cells, such as interstitial fibrosis, affecting the compliance of the heart; and myocyte loss, unmatched by myocyte replacement. Cardiac regeneration is robust in certain organisms, such as the newt and zebra fish, where total replacement can transpire, via the production of an undifferentiated cell mass, called the blastema. ${ }^{1}$ The regeneration of adult cardiomyocytes is impossible in mammals under normal, unassisted biological circumstances. Several complementary strategies can be helpful in potentially aiding this process: over riding cellcycle checkpoints that constrain the reactive proliferation of ventricular myocytes; ${ }^{2}$ supplementing the naturally 
occurring cytoprotective mechanisms, or inhibiting prodeath pathways; ${ }^{3}$ supplementing the naturally occurring angiogenic mechanisms using defined growth factors or arteriole-forming cells; ${ }^{4}$ or providing exogenous cells as a surrogate or precursor for cardiac muscle itself. ${ }^{5}$ Among these conceptual possibilities, cell transplantation, in various forms, is the first strategy that has been translated from the bench to the bedside. Although there is increasing evidence that the heart can renew itself by activation of resident cardiac stem cells, this endogenous capacity for regeneration is insufficient to mediate repair after severe cardiac injury. ${ }^{6}$ As shown in recent clinical trials, exogenous delivery of stem cells to injured areas of the myocardium may overcome this limitation.

Stem cells are traditionally isolated from bone marrow and demonstrate excellent safety in clinical practice. But repair outcome and patient-to patient variability in heart failure patients are yet to establish.

In this point of view the present clinical trial is undertaken to assess the effect of stem cell therapy in patients with heart failure in our setting.

\section{Methodology:}

This pilot study was carried out at the Department of cardiology in collaboration with department of haematology, Bangabandhu Sheikh Mujib Medical University, Dhaka, from October 2017 to March 2018. The research protocol was approved by the Institutional Review Board, BSMMU, Dhaka. Patients with chronic heart failure of ischemic origin were screened, and patients of 18 to 60 years of age, both sexes, having $\mathrm{H} / \mathrm{O}$ ischemic event at least 2 months before recruitment, impaired left ventricular ejection fraction (LVEF $15 \%$ to $40 \%$ ) and getting treatment according to guidelines (GDMT) including revasculrization and implantable cardioverter-defibrilator as needed were enrolled as study population. Patients having ventricular aneurysm, moderate to severe aortic stenosis, left ventricular thrombus and co-morbidity like any malignancy, uncontrolled diabetes, respiratory diseases, hepatic or renal impairment, any evidence of concurrent sepsis and previous history of cell therapy were excluded. Ten (10) patients were taken in stem cell group and ten (10) patients in control group.

A written informed consent for inclusion in the study was obtained from all patients. The potential benefits and risks of the use of stem cell therapy as well as the non-availability of the heart transplant facilities was explained to them. Baseline data including age, sex, body mass index, presence of cardiovascular risk factors, and meticulous cardiac disease history was taken. Patients in the control arm received standard of care comprising a beta-blocker, an angiotensin-converting enzyme inhibitor or angiotensin receptor blocker \& a diuretic with dosing schedule tailored for maximal benefit and tolerability in accordance with practice guidelines for heart failure management (GDMT). Patients in the cell therapy arm received, in addition to standard of care, bone marrow-derived cardiopoietic stem cells (G-CSF) meeting quality release criteria. Baseline clinical and echocardiographic data were obtained and recorded in pre-formed data sheet.

All patients were treated under Department of Cardiology, BSMMU. Patients of control group were treated in OPD \& in-door basis as per clinical need. And patients of study group were admitted in dept of cardiology, BSMMU and received G-CSF (Injection Grastim; Square Pharmaceuticals Ltd., Bangladesh) at a dose of 300 IU S/C daily for five days, along with standard medical therapy. Then $50 \mathrm{ml}$ venous blood was obtained from peripheral veins of the study subjects maintaining all aseptic precautions. Stem cells were isolated from peripheral blood using Cell Sorter (Fresenius Kabi, Germany) at the Department of Haematology and Pathology BSMMU. Subsequently coronary artery of the study subjects was selectively catheterized in 'Cath lab' at the Department of Cardiology, BSMMU and isolated stem cells with anti-coagulant (Injection Heparin) was injected. During the injection, a balloon was kept inflated in coronary artery, proximally, for one minute, in order to prevent washout of the cells. The entire procedure from collection of peripheral venous blood, isolation of stem cells and injection of stem cells into the heart was done on the same day within the shortest possible time.

Close liaison was maintained with all patients. Permanent address, present address and cell/phone number of all patients were kept and patients were followed up after 30 days $\&$ after 3 months and for any complication.

All patients were advised to contact immediately if any need arises. Each patient was advised for follow up according to schedule. During follow up period, in case any patient is admitted to any local hospital for detoriation and/or death, inquiry about the patient's condition and cause of death was gathered from the attending physician at that particular hospital.

During follow up clinical and echocardiographic data was taken and recorded in preformed data collection sheet.

Patient's clinical data was taken from history $\&$ clinical examination. Objective evidence of functional capacity \& clinical improvement was assessed by 6 minutes walk test (6MWT).

Echocardiography was done with Vivid 9 echocardiography machine, by SIEMENS, at echocardiography laboratory of the Department of Cardiology, BSMMU.

\section{Statistical processing and analysis}

Quantitative data was presented as mean \pm SD and qualitative data in percentage. Chi-square test was used 
to analyze intra \& inter group comparison between study and control groups. A statistically significant result was considered when $p$ values will beless than 0.05 . All data were analyzed by SPSS (version 20).

\section{Results \& observations}

The present study intended to investigate the effects of stem cell therapy in patients with ischemic heart failure. The findings obtained from data analysis are presented below:

Total study populations were $20.50 \%(\mathrm{~N}=10)$ patients were in control group (GDMT only) \& 50\% $(\mathrm{N}=10)$ patients were in stem cell therapy group (SCT + GDMT)

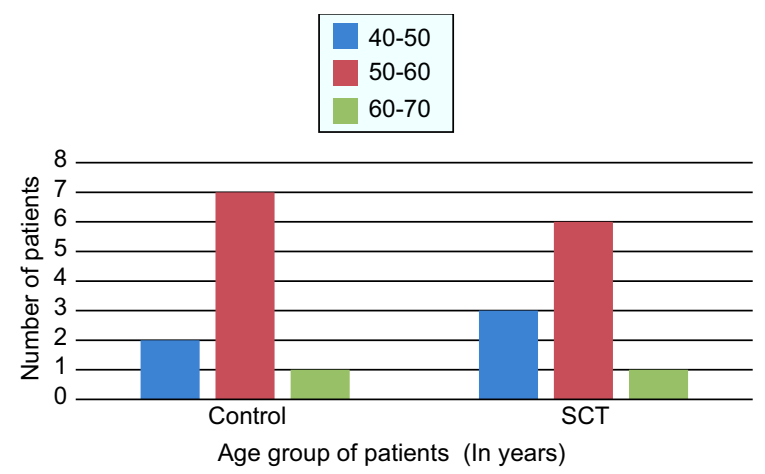

Fig.-1: Age distribution of the study population $(n=20)$

The above figure shows age distribution of study population. Majority of patients were in the age range of $50-60$ years in both control $(n=7) \&$ SCT group $(n=6)$. The mean ages of control group was $54 \pm 6$ years and SCT group was $55 \pm 54$ years.
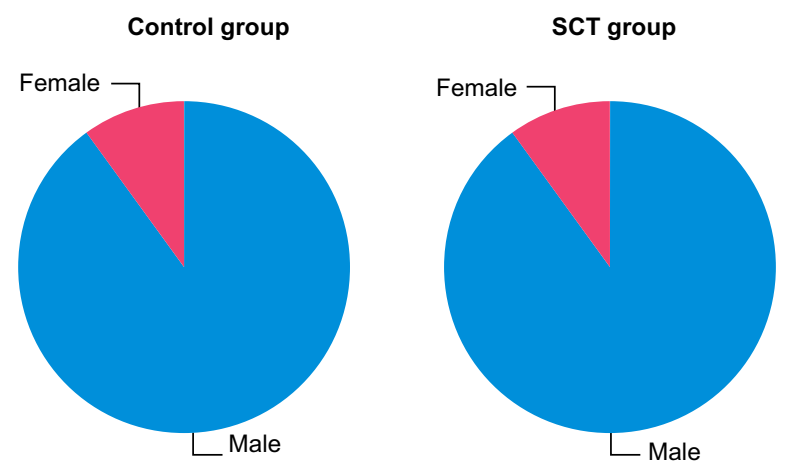

Fig.-2: Sex distribution of study population $(n=20)$

The above figure shows sex distribution among the study population. In both group, $90 \%$ were male \& $10 \%$ were female.

The above table shows distribution of risk factors of the study population. Considering risk factors, DM had high percentage in both groups (Control group, $100 \%$ and SCT, 90\%) which was not statistically significant $(p>0.05)$. Hypertension were found $60 \%$ \& $70 \%$ respectively in control and SCT group which was not statistically significant $(p>0.05)$. Dyslipidemia was present in $70 \%$ of patients in both groups. No statistically significant difference was observed in term of dyslipidemia of both groups $(\mathrm{p}>0.05)$.

The above table shows pattern of infarct area involvement among the study population. In control group, all patients were OMI (100\%) whereas $90 \%$ patients were OMI in SCT group. Anterior MI had the highest percentage (50\% \& $70 \%$ respectively) followed by Extensive Anterior MI (40\% $\& 20 \%$ respectively) in both groups.

There was no significant difference at baseline 6MWD between the two groups (175 \pm 20.17 in the control group and $142 \pm 32.25$ in the SCT group, $p=0.12$ ). The absolute change in 6MWD from baseline to 30 days, 3 months \& 6 months improved significantly in the both groups. But significant improvement was found at 6 months follow up of 6MWD between the two groups ( $300 \pm 28$ vs $375 \pm 25, \mathrm{p}=0.04$ ).

Baseline $\mathrm{SPO}_{2}$ was similar in the control group and the SCT group which was not statistically significant $(89 \pm 0.84$ and $88 \pm 0.84$, respectively, $\mathrm{P}=0.12$ ). During follow up at 30 days, $\mathrm{SPO}_{2}$ was improved similarly in both group. Follow up observation at 3 months \& 6 months revealed a significant increase in $\mathrm{SPO}_{2}$ from baseline to $3 \& 6$ months respectively in both groups but in between group, SPO2 improvement was statistically significant at only 6 months follow up $(94 \pm 0.7 \%$ and $96 \pm 0.8 \%, \mathrm{P}=0.04)$.

Baseline BORG scale was similar in the control group and the SCT group $(8.1 \pm 0.56$ and $8.3 \pm 0.67$ respectively, $\mathrm{P}=0.45)$. The absolute change in BORG scale from baseline to 30 days, 3 months \& 6 months improved significantly in the both groups. But improvement was not statistically significant in between the two groups $(p=0.32,0.45,0.23$ respectively).

Echocardiographic observation also revealed a similar baseline LVIDd, LVEF level in the control group and the SCT group which was not statistically significant $(\mathrm{p}=$ $0.45,0.52$ respectively). Gradual improvement in LVIDd were found at 30 days, 3 months, 6 months follow up observation but statistically significant absolute change was found only at 6 months follow up in between groups $(62.4 \pm 1.8$ vs $56 \pm 2.4, \mathrm{p}=0.03)$. Baseline LVEF were less than $30 \%$ in both control \& SCT group $(29.5 \pm 0.8 \%$ \& $28.7 \pm 1.3 \%$ respectively). The echocardiographic evaluation also revealed a significant increase in LVEF at 6 months $(34 \%$ \pm 1.6 and $40 \% \pm 2.5 \%, p=0.04)$ of follow-up in between group but not at 30 days \& 3 months follow up.

All procedures related to SCs transplantation were well tolerated. Patients had no or mild angina during balloon inflation for infusion of SCs. There were no serious 
Table-I

Distribution of risk factors of the study population $(n=284)$

\begin{tabular}{|c|c|c|c|c|c|c|}
\hline \multirow[t]{2}{*}{ Risk factors } & & \multicolumn{2}{|c|}{ Control $(n=10)$} & \multicolumn{2}{|c|}{$\mathrm{SCT}(\mathrm{n}=10)$} & \multirow[t]{2}{*}{ p-value } \\
\hline & & Number & $\%$ & Number & $\%$ & \\
\hline \multirow[t]{2}{*}{$\overline{\mathrm{HTN}}$} & Yes & 06 & 60 & 07 & 70 & $0.8^{\mathrm{NS}}$ \\
\hline & No & 04 & 40 & 03 & 30 & \\
\hline \multirow[t]{2}{*}{$\mathrm{DM}$} & Yes & 10 & 100 & 09 & 90 & $0.9^{\mathrm{NS}}$ \\
\hline & No & 0 & 00 & 01 & 10 & \\
\hline \multirow[t]{2}{*}{ Dyslipidemia } & Yes & 07 & 70 & 07 & 70 & $1.0^{\mathrm{NS}}$ \\
\hline & No & 03 & 30 & 03 & 30 & \\
\hline
\end{tabular}

Table-II

Pattern of infarct area of ICM among the study group $(n=20)$

\begin{tabular}{lccccc}
\hline ICM & \multicolumn{2}{c}{ Control $(\mathrm{n}=10)$} & \multicolumn{2}{c}{ SCT $(\mathrm{n}=10)$} & $\mathrm{p}$-value \\
& Number & $\%$ & Number & $\%$ & \\
\hline OMI & 10 & 100 & 09 & 90 & 0.9 \\
Anterior & 05 & 50 & 07 & 70 & 0.8 \\
Inferior & 01 & 10 & 01 & 10 & 0.01 \\
Ext. Anterior & 04 & 40 & 02 & 20 & 0.5 \\
\hline
\end{tabular}

\section{Table-III}

Quantitative analysis of outcome in terms of $6 M W D(n=20)$

\begin{tabular}{lccc}
\hline 6MWD & $\begin{array}{c}\text { Control }(\mathrm{n}=10) \\
(\text { Mean } \pm \text { SD) }\end{array}$ & $\begin{array}{c}\text { SCT }(\mathrm{n}=10) \\
(\text { Mean } \pm \text { SD) }\end{array}$ & p-value \\
\hline At Baseline & $175 \pm 20.17$ & $142 \pm 32.25$ & 0.12 \\
30 days & $221 \pm 16.63$ & $210 \pm 24.94$ & 0.5 \\
3 month & $264 \pm 30.62$ & $267 \pm 52.29$ & 0.4 \\
6 month & $300 \pm 28$ & $375 \pm 25$ & 0.04 \\
P value (30 days Vs Baseline) & 0.01 & 0.03 & \\
P value (3month Vs Baseline) & 0.003 & 0.002 & \\
P value (6month Vs Baseline) & 0.01 & 0.02 & \\
\hline
\end{tabular}

Table-IV

Quantitative analysis of outcome in terms of $\mathrm{SPO}_{2}(n=20)$

\begin{tabular}{lccc}
\hline $\mathrm{SPO}_{2}$ & $\begin{array}{c}\text { Control }(\mathrm{n}=10) \\
(\mathrm{Mean} \pm \mathrm{SD})\end{array}$ & $\begin{array}{c}\mathrm{SCT}(\mathrm{n}=10) \\
(\text { Mean } \pm \mathrm{SD})\end{array}$ & $\mathrm{p}$-Value \\
\hline At Baseline & $89 \pm 0.84$ & $88 \pm 0.84$ & 0.12 \\
30 days & $91 \pm 0.64$ & $91 \pm 1.03$ & 0.7 \\
P value (30 days Vs Baseline) & 0.2 & 0.4 & 0.4 \\
3 month & $93 \pm 0.5$ & $94 \pm 0.94$ & \\
P value (3 month Vs Baseline) & 0.03 & 0.02 & 0.04 \\
6 month & $94 \pm 0.7$ & $96 \pm 0.8$ & \\
P value (6month vs Baseline) & 0.02 & 0.01 & \\
\hline
\end{tabular}

procedural complications related to intracoronary administration of the stem cell, such as ventricular arrhythmias, peri-procedural MI, thrombus formation or dissection. There were no deaths, rehospitalization, MI, stent thrombosis, life-threatening arrhythmia, or stroke in both groups up to 6 months follow-up. 
Table-V

Quantitative analysis of outcome in terms of BORG scale $(n=20)$

\begin{tabular}{lccc}
\hline BORG scale & $\begin{array}{c}\text { Control }(\mathrm{n}=10) \\
(\text { Mean } \pm \text { SD) }\end{array}$ & $\begin{array}{c}\text { SCT }(\mathrm{n}=10) \\
(\text { Mean } \pm \text { SD })\end{array}$ & p-Value \\
\hline At Baseline & $8.1 \pm 0.56$ & $8.3 \pm 0.67$ & 0.45 \\
30 days & $6.8 \pm 0.63$ & $6.9 \pm 0.56$ & 0.32 \\
3 month & $5.3 \pm 0.48$ & $5.4 \pm 0.51$ & 0.45 \\
6 month & $3.6 \pm 0.69$ & $3.0 \pm 0.23$ & 0.23 \\
P value(30 day vs Baseline) & 0.03 & 0.04 & \\
P value (3 month vs Baseline) & 0.02 & 0.03 & \\
P value (6month vs Baseline) & 0.01 & 0.01 & \\
\hline
\end{tabular}

Table-V

Quantitative analysis of LV function by echocardiography $(n=20)$

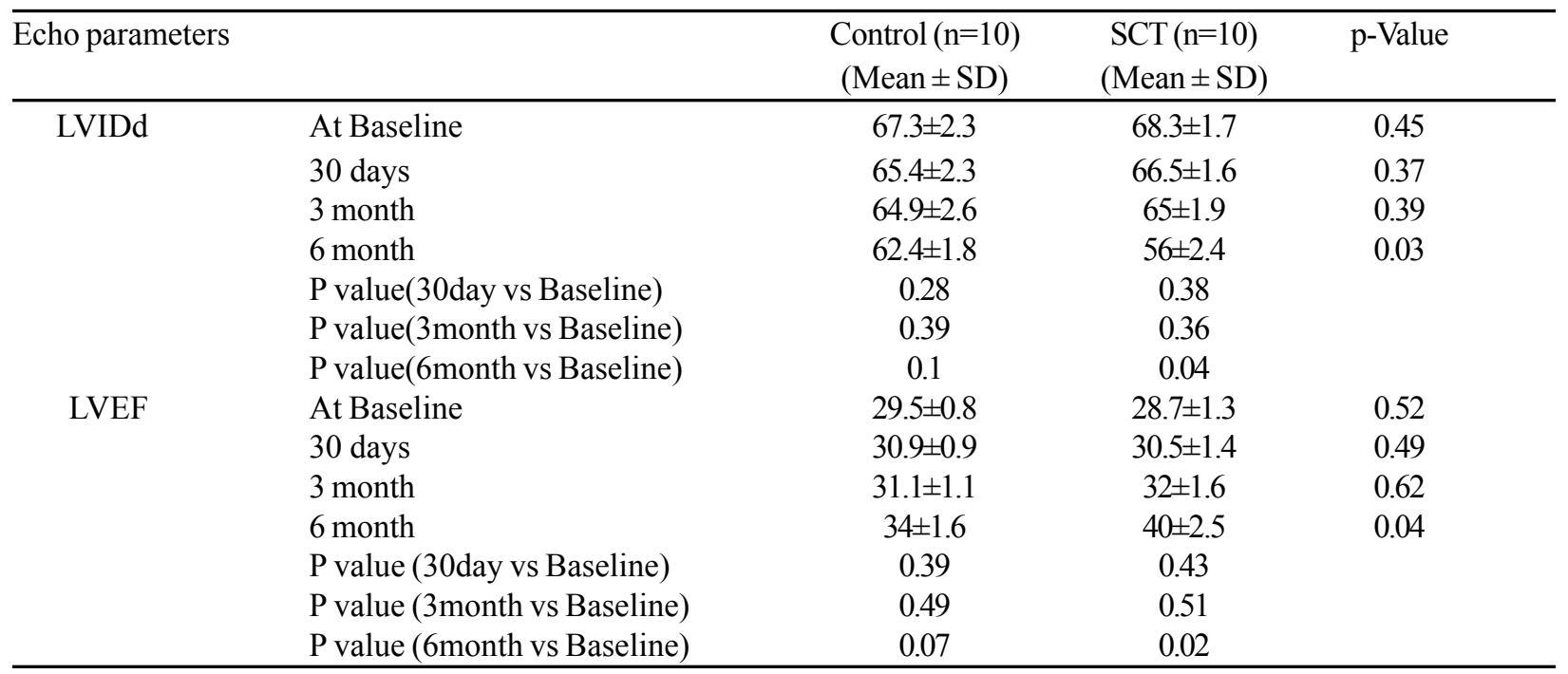

\section{Discusion:}

In patients with ischemic heart failure, progressive myocyte loss from persistent and uncorrected ischemia, sustained inflammation processes and apoptosis result in worsening fibrosis and replacement of functioning myocardium with scar thereby resulting in systolic dysfunction, myocardial remodeling, and finally, heart failure. Since its discovery, stem cell therapy (SCT) has become a new treatment strategy in patients with advanced ischemic heart failure to improve cardiac function. ${ }^{7}$ The present study was carried out with an aim to investigate the efficacy of SCT along with GDMT in ischemic heart failure patient with no other revascularization option. In our study total 20 ischaemic heart failure patient were included \& grouped into SCT group who were treated with stem cells along with GDMT and control group who were treated with only GDMT, not amenable to revascularization. Both groups were followed up upto 6 months by $6 \mathrm{MWD}$, BORG scale, LVEF by echocardiography.
Though the mechanism of action of stem cell therapy is debatable but commonly attributed mechanism of stem cell therapy is the regeneration of heart cells, leading to a restored, functioning myocardium. However, the immediate cardioprotective effects of stem cells within one day of administration is questionable. ${ }^{8}$ These facts conclude that the effects of stem cells cannot be solely due to regeneration. Thus, these effects could be due to some paracrine effects of stem cells, such as increasing cell proliferation, stimulated cell recovery, apoptosis prevention, and promoting healing. ${ }^{9-12}$ As paracrine effects can improve over time, a longer follow-up is essential after stem cell transplantation. In our study we followed up our patients up to 6 months. Perin et al., ${ }^{13}$ Silva et al. ${ }^{14}$ conducted similar study where they followed up 4 months $\& 6$ months respectively.

In the present study, mean age of total study population was $54.58 \pm 56$ years. The commonest age group of study patients was 50-60 years. In both group, $90 \%$ were male which is similar to Florea et al. study. ${ }^{15}$ So, like other 
studies, males were predominant in our study. Among risk factors, DM, hypertension \& dyslipidemia were found statistically non significant among the study groups. Commonest risk factor was DM in both groups $(100 \%$ \& $90 \%$ in control \& SCT group respectively).

Although various parameters were followed up in different studies \& clinical trials including follow-up time, the number of cells, cell type that was transplanted to ischemic myocardium and route of administration. Most of the studies and trials used bone marrow mononuclear cells for transplantation to repair the injured heart myocardium. ${ }^{16}$ There are many other sources for stem cells, such as tissue-derived, embryonic-derived and reprogrammed cells, which are still under investigation. Also, there are many routes of administration of stem cells such as intracoronary, intravenous infusion, epicardial, or transendocardial injection via a catheter (Intramyocardial). ${ }^{17}$ In our study, we used autologus bone marrow derived SCs which was delivered by Intracoronary route into infarct related artery. In terms of safety of route of administration, intracoronary administration of SCs showed no serious adverse events like periprocedural MI, arrhythmia in our study. It seems to be a safe method to de-liver stem cells via intracoronary route, since its introduction by Strauer et al. ${ }^{18}$ Moreover, ischemic preconditioning in-duced by transient balloon occlusion seems to be important to recruit SCs into the infarcted myocardium. ${ }^{19,20}$

This study demonstrated a significant improvement in 6MWD, $\mathrm{SpO}_{2}$, BORG scale, LVEF by echocardiography with no effect on mortality in SCT group compared to control group. Though absolute improvement in 6MWD from baseline to 3 months \& 6 months was found in the both groups. But statistical significant improvement was found at 6 months follow up of 6MWD between the two groups $(300 \pm 28$ vs $375 \pm 25, \mathrm{p}=0.04)$. Similar result was found in terms of $\mathrm{SpO}_{2}$. IHF symptoms \& exercise intolerance, as indicated by the BORG scale, significantly decreased with SCT. Overall, SCT has been shown to be safe as a treatment for IHF with no increase in mortality. In chronic ischemic heart failure, STAR-Heart study, a largest clinical trial of intracoronary autologous mononuclear BMC transplantation demonstrated that intracoronary BM cell therapy improved ventricular performance (LVEF), qualityof-life and even survival. ${ }^{21}$ The echocardiographic evaluation of our study also revealed a significant increase in LVEF at 6 months follow up in both control and SCT group ( $29.5 \pm 0.8$ to $34 \pm 1.6 \%$ and $28.7 \pm 1.3$ to $40 \pm 2.5 \%$ respectively, $\mathrm{p}=0.04$ ). But SCT group showed more significant improvement at 6 months from the baseline than GDMT only group $(\mathrm{p}=0.02$ vs $\mathrm{p}=0.07)$. Using intracoronary injection of BMCs, Strauer et al. ${ }^{22}$ reported a $30 \%$ reduction in infarct size, a $15 \%$ increase in global ejection fraction and a $57 \%$ increase in infarction zone wall motion-determined left ventriculography.

Friis et al. ${ }^{23}$ conducted a safety study and enrolled 31 patients with stable, moderate-severe angina with no further revascularization options. MSCs (mean: $21.5 \times 10^{6}$, range $3-62 \times 10^{6}$ ) were delivered by intramyocardial injection, and all recipients were followed for 6 months. SPECT analysis showed no difference in the perfusion score, and cardiac MRI showed improvement in LVEF from $55.9 \%$ to $57.9 \%(\mathrm{p}<.001)$. There was also an improvement in exercise capacity and Canadian Cardiovascular Society (CCS) class of angina, although these results were not placebo-controlled. There were no major adverse cardiac events (MACE) associated with the stem cells. In 2015, from the results of the MSC-HF trial, a randomized controlled trial for patients with symptomatic ischemic cardiomyopathy $(\mathrm{LVEF}<45 \%)$ Mathiasen et al. ${ }^{24}$ demonstrated that there were significant improvements in LVEF of $6.2 \%(p<.0001)$, stroke volume of $18.4 \mathrm{ml}(\mathrm{p}<.0001)$, and myocardial mass of $5.7 \mathrm{~g}(\mathrm{p}=.001)$ in MSC group compared to placebo at 6 months of follow-up. Sixty patients were enrolled and MSC group received a mean of $8.3 \times 10^{7}$ autologous BM cells.

Though more and more medical centers regard SCT as a promising treatment for IHF, which is caused by myocardial remodeling after AMI. But meanwhile, the safety and clinical efficacy of SCT as a treatment for IHF is still controversial and requires further evaluation in clinical trials. ${ }^{16}$ There are some other questionable point of views that may be noteworthy like route of cell administration or delivery method, source of stem cells, amount of cells, timing of cell administration. One meta-analysis showed that cell number was an independent predictor of outcome on LV function, with trials using greater than $50 \times 10^{6}$ cells having more efficacy than those using less. ${ }^{25}$ Regardless, the data for BM-derived cells overall show a small but significant benefit in LVEF (12.92\%), reduction in infarct size $(22.25 \%)$, and LVESV $(26.37 \mathrm{ml})$ compared with standard therapy. ${ }^{25}$ In our study, the intracoronary admin-istration of autologous purified BM-derived SCs at least 2 months after ischaemic attack is well tolerable without serious complications \& mortality and pro-vides modest improvement in LVEF, $\mathrm{O}_{2}$ consumption, symptoms at 6-month follow-up in SCT group.

Our study has several limitations. First, our study is a pilot study and enrolled relatively small number of patients. This limitation may attenuate the efficacy of intracoronary purified MSCs. Further large-scale randomized trials are needed. Second, there may be a technical problem for the assessment of LVEF. Although CMR was considered to be the "gold standard" for evaluation of LV function, it was impossible to use CMR at all institutions for the first time. Third, we did not use di-verse assessment tools such 
as the NYHA, CCS, ex-ercise tolerance, pulmonary function test and quality of life. Finally, we did not evaluate the inter and intra-observer variability in radiographic measurements. Our study group showed a moderate improvement of LV systolic function and low risk profiles. Long-term follow-up is needed to define the safety and beneficial effect of MSCs.

In summary, this pilot study was designed to identify the efficacy of intracoronary purified autologous BM-derived SCs in patients with ishaemic heart failure with no other revascularization option. Intracoronary administration of autologous BM-derived SCs at least 2 months after ischaemic attack is well tolerable and safe with modest improvement in exercise capacity, LVEF at 6-month follow-up.

\section{References:}

1. Poss KD, Wilson LG, Keating MT. Heart regeneration in zebrafish. Science 2002;298:2188-90.

2. Pasumarthi KB, Nakajima H, Nakajima HO, Soonpaa MH, Field LJ. Targeted expression of cyclin D2 results in cardiomyocyte DNA synthesis and infarct regression in transgenic mice.Circ Res 2005;96:110-8.

3. Oh H, Wang SC, Prahash A, et al. Telomere attrition and chk2 activation in human heart failure. ProcNatlAcadSci USA $2003 ; 100: 5378-83$.

4. Losordo DW, Dimmeler S. Therapeutic angiogenesis and vasculogenesis for ischemic disease:part I. angiogenic cytokines. Circulation 2004;109:2487-91. 5) Olson EN, Schneider MD. Sizing up the heart:developmentredux in disease. Genes Dev 2003;17:1937-56.

5. Olson EN, Schneider MD. Sizing up the heart: developmentredux in disease. Genes Dev 2003;17:1937-56. http://genesdev.cshlp.org/lookup/doi/10.1101/gad.1110103

6. Smith RR, Barile L, Cho HC, Leppo MK, Hare JM, MessinaE, et al. Regenerative potential of cardiosphere-derived cellsexpanded from percutaneous endomyocardial biopsy specimens.Circulation. 2007 Feb 20;115:896-908.

7. Hu S, Liu S, Zheng Z, Yuan X, Li L, Lu M, Shen R, Duan F, Zhang X, Li J, Liu X, Song Y, Wang W, Zhao S, He Z, Zhang $\mathrm{H}$, Yang $\mathrm{K}$, Feng W, Wang X. Isolated coronary artery bypass graft combined with bone marrow mononuclear cells delivered through a graft vessel for patients with previous myocardial infarction and chronic heart failure: a single-center, randomized, double-blind, placebo-controlled clinical trial. $J$ Am Coll Cardiol. 2011;57:2409-15.

8. Kupatt C, Hinkel R, Lamparter M, et al.: Retroinfusion of embryonic endothelial progenitor cells attenuates ischemiareperfusion injury in pigs. Role of phosphatidylinositol 3kinase/AKT kinase. Circulation. 2005, 112:117-22.

9. Kupatt C, Horstkotte J, Vlastos GA, et al.: Embryonic endothelial progenitor cells expressing a broad range of proangiogenic and remodeling factors enhance vascularization and tissue recovery in acute and chronic ischemia. FASEB J. 2005, 19:1576-78.

10. Gnecchi M, Zhang Z, Ni A, Dzau VJ: Paracrine mechanisms in adult stem cell signaling and therapy. Circ Res. 2008, 103:1204-19.
11. Penn MS, Dong F, Klein S, Mayorga M: Stem cells for myocardial regeneration . Clin Pharmacol Ther. 2011, 90: 499-501.

12. Uemura R, Xu M, Ahmad N, Ashraf M: Bone marrow stem cells prevent left ventricular remodeling of ischemic heart through paracrine signaling. Circ Res. 2006, 98:1414-21.

13. Perin EC, Dohmann HF, Borojevic R, et al. Transendocardial, autologous bone marrow cell transplantation for severe, chronic ischemic heart failure. Circulation 2003;107: 2294-302.

14. Silva GV, Perin EC, Dohmann HF, et al. Catheter-based transendocardial delivery of autologous bone-marrow-derived mononuclear cells in patients listed for heart transplantation. Tex Heart Inst J 2004;31:214-19.

15. Florea V, Rieger AC, DiFede DL, et al.: Dose comparison study of allogeneic mesenchymal stem cells in patients with ischemic cardiomyopathy (the TRIDENT study). Circ Res. 2017, 10:1279-90.

16. Yixuan Wang, Fen Xu, Jingwei Ma, Jiawei Shi, Si Chen, Zongtao Liu, Junwei Liu. Effect of stem cell transplantation on patients with ischemic heart failure: a systematic review and meta analysis of randomized controlled trials. Stem Cell Research \& Therapy, 2019;10:125.

17. Jezierska-Wozniak K, Mystkowska D, Tutas A, Jurkowski MK: Stem cells as therapy for cardiac disease - a review. Folia Histochem Cytobiol. 2011, 49:13-25.

18. Strauer BE, Brehm M, Zeus T, Köstering M, Hernandez A, Sorg $\mathrm{RV}, \mathrm{Kö}-\mathrm{gler} \mathrm{G}$, Wernet P. Repair of infarcted myocardium by autologous intra-coronary mononuclear bone marrow cell transplantation in humans. Circulation 2002; 106: 1913-8.

19. Lu G, Haider HK, Jiang S, Ashraf M. Sca-1+ stem cell survival and en-graftment in the infarcted heart: dual role for preconditioning-induced connexin-43. Circulation 2009; 119: 2587-96.

20. Kamota T, Li TS, Morikage N, Murakami M, Ohshima M, Kubo M, Ko-bayashi T, Mikamo A, Ikeda Y, Matsuzaki M, et al. Ischemic pre-condi-tioning enhances the mobilization and recruitment of bone marrow stem cells to protect against ischemia/reperfusion injury in the late phase. J Am Coll Cardiol 2009; 53: 1814-22.

21. Strauer BE, Yousef M, Schannwell CM. The acute and longterm effects of intracoronary Stem cell Transplantation in 191 patients with chronic heARt failure: the STAR-heart study. Eur J Heart Fail 2010; 12: 721-9.

22. Strauer BE, Brehm M, Zeus T, et al. Regeneration of human infarcted heart muscle by intracoronary autologous bone marrow cell transplantation in chronic coronary artery disease: the IACT Study. J Am Coll Cardiol 2005;46:1651-8

23. Friis $T$, Haack-Sorensen $M$, Mathiasen $A B$ et al. Mesenchymal stromal cell derived endothelial progenitor treatment in patients with refractory angina. Scand Cardiovasc J 2011;45:161-68.

24. Mathiasen AB, Qayyum AA, Jorgensen E et al. Bone marrowderived mesenchymal stromal cell treatment in patients with severe ischaemic heart failure: A randomized placebo-controlled trial (MSC-HF trial). Eur Heart J 2015;36:1744-53.

25. Afzal MR, Samanta A, Shah ZI et al. Adult bone marrow cell therapy for ischemic heart disease: Evidence and insights from randomized controlled trials. Circ Res 2015; 117:558-75. 